\title{
DINÁMICA DE LA CUERDA BOSÓNICA CIRCULAR
}

\author{
Fulgencio Villegas Silva ${ }^{a^{*},}$ Rene Negrón Huamán ${ }^{a}$, Manuel Culqui Rodríguez ${ }^{\mathrm{a}}$ \\ ${ }^{a}$ Facultad de Ciencias Físicas, Universidad Nacional Mayor de San Marcos Ap. Postal 14-0149, Lima 14, Perú.
}

(Recibido Abril 28, 2009; Aceptado Julio 20, 2009)

\begin{abstract}
Resumen
Luego de una breve discusión sobre la acción de Nambu-Goto, se introduce el concepto de velocidad transversal y se encuentra dicha velocidad para una cuerda bosónica circular cerrada, luego se estudian y resuelven las ecuaciones de movimiento de la cuerda haciendo uso de la ley de conservación de la energía y finalmente esbozamos su correspondiente hoja de mundo.
\end{abstract}

PACS: 04.60.Cf; 11.25.Pm

Palabras claves: Teoría de cuerdas, cuerdas bosónicas, teoría de supercuerdas.

\begin{abstract}
After a brief discussion about the Nambu-Goto action, we introduce the concept of transverse velocity and find it for a closed circular bosonic string, then we study and solve the string's equations of movement by using the energy conservation law and finally we sketch its corresponding world sheet.
\end{abstract}

Keywords: string theory, bosonic string, superstring theory.

\section{Introducción}

En la última década del siglo pasado, uno de los mayores problemas de la física de altas energías consistía en encontrar una descripción consistente para el mundo a escala sub-atómica. En las últimas décadas, se puede decir, que las teorías que han tenido más preponderancia en la física teórica son la teoría cuántica de campos y la relatividad general. Sin embargo, estas teorías no se complementan cuando se las aplica al mismo ámbito de la física. En este contexto, la teoría que contiene las mejores características para la unificación de todas las fuerzas es la teoría de cuerdas que por ende lograría la unión de la teoría gravitacional de Einstein y la Mecánica Cuántica.

La teoría de cuerdas es una teoría cuántica en la cual los constituyentes fundamentales del universo son objetos matemáticos extensos, unidimensionales, descritos en un espacio-tiempo multidimensional.

La teoría de cuerdas bosónicas es la versión original de la llamada teoría de supercuerdas (abreviado, teoría de cuerdas). Esta es una teoría de cuerdas relativista, y cuya principal característica es que al ser cuantizada, esta solo presenta estados bosónicos, por lo cual se puede decir que predice solo la existencia de bosones (he ahí el porque de su nombre). Los estados fermiónicos se presentan cuando se incluye la supersimetría dentro la teoría. Los físicos han calculado que, para su consistencia, la teoría de cuerdas bosónicas requiere de 26 dimensiones espacio-temporales [1,2].

Y. Nambu y T. Goto [3,4] propusieron que la acción mas simple para una cuerda bosónica seria proporcional al área descrita por la cuerda cuando ella evoluciona en el espacio-tiempo, de donde se deduce que la acción de Nambu-Goto presenta la forma.

$S=-\frac{T_{0}}{c} \int d \tau d \sigma \sqrt{\left(\dot{X} \cdot X^{\prime}\right)^{2}-(\dot{X})^{2}\left(X^{\prime}\right)^{2}}$,

donde $T_{0}$ es la llamada tensión de la cuerda, $T_{0}=\frac{1}{2 \pi \alpha^{\prime}}, \alpha^{\prime}$ es la inclinación de Regge, siendo $\alpha^{\prime}=\ell_{C}^{2}$, donde $\ell_{C}$ es la longitud de la cuerda.

La existencia de la raíz cuadrada en la acción de Nambu-Goto (1) introduce dificultades en el cálculo, utilizando una métrica intrínseca auxiliar [5,6] de la hoja de mundo obtenemos una acción equivalente, llamada acción de Polyakov [7] dada por:

\footnotetext{
*Correspondig author.e-mail: fvillegass@unmsm.edu.pe
} 
$S_{P}=-\frac{T_{0}}{2 c} \sqrt{g} g^{a b} \eta_{\mu \nu} \partial_{a} X^{\mu} \partial_{b} X^{v}$

Las cuerdas bosónicas pueden ser abiertas o cerradas. Así como en la cuerda bosónica abierta los campos gauge ocurren naturalmente, en la cuerda bosónica cerrada, el campo gravitacional ocurre naturalmente. Resulta, en efecto, que un campo gravitacional, un campo dilatónico y un campo tensorial antisimétrico ocurren en la cuerda cerrada en la misma forma que el campo gauge aparece en la cuerda abierta. El estado fundamental $|0\rangle$ de la cuerda cerrada es un taquión. Los primeros estados excitados son estados másicos de la forma $X_{+}^{\mu}(\sigma) X_{+}^{v}(\sigma)|0\rangle$ que son la parte simétrica, la traza y la parte antisimétrica de un tensor formado por las $X$ que son identificados con el campo gravitacional, el dilatón y un tensor de campo antisimétrico respectivamente [8].

En este trabajo estudiamos la dinámica de la cuerda bosónica circular. Este trabajo fue inspirado en el problema 6.4 del libro: A first course in string theory, Barton Zwiebach, Cambridge University Press, 2004

\section{Velocidad transversal}

Existe una velocidad invariante que se puede definir en la cuerda, esta es sin embargo una velocidad transversal. Considerando el movimiento espacial de la cuerda, se puede considerar que cada punto en la cuerda se mueve transversalmente a ella. La velocidad transversal $\vec{v}$ en cada punto de la cuerda es un vector ortogonal a la misma y tangente a la superficie espacial de la cuerda [9], esto es:

$\vec{v}=\frac{\partial \vec{X}}{\partial t}-\left(\frac{\partial \vec{X}}{\partial t} \cdot \frac{\partial \vec{X}}{\partial s}\right) \frac{\partial \vec{X}}{\partial s}$

Además $X^{\mu}$ son coordenadas espaciotemporales de la cuerda, donde $X^{\mu}=\left\{X^{0}, \vec{X}\right\}$.

Para el caso más simple de cuerda bosónica cerrada, que corresponde a una del tipo circular, la magnitud de la velocidad transversal no es más que la razón de cambio en el tiempo de su radio

$|\vec{v}|=\left|\frac{\partial \vec{X}}{\partial t}\right|=\frac{d R}{d t}=\dot{R}$
La ecuación (4) resulta de tomar el módulo de la ecuación (3), considerando el vector $\vec{X}$ en coordenadas polares donde $d s=R d \theta$.

Ahora concentraremos nuestra atención en escribir la acción (1) en términos de la velocidad transversal antes descrita.

Podemos escribir las coordenadas $X^{\mu}$ de la siguiente manera:

$X^{\mu}(\tau, \sigma)=X^{\mu}(t, \sigma)=\{c t, \vec{X}(t, \sigma)\}$,

donde se ha considerado el gauge estático [9]: $X^{0}(\tau, \sigma) \equiv c t(\tau, \sigma)=c \tau$.

Usando la ecuación (5) y la definición (3), podemos calcular los productos punto relativistas que aparecen en la ecuación (1), de esta manera:

$$
\begin{aligned}
& (\dot{X})^{2}=-c^{2}+\left(\frac{\partial \vec{X}}{\partial t}\right)^{2}, \\
& \left(X^{\prime}\right)^{2}=\left(\frac{\partial \vec{X}}{\partial \sigma}\right)^{2}, \\
& \dot{X} \cdot X^{\prime}=\frac{\partial \vec{X}}{\partial t} \cdot \frac{\partial \vec{X}}{\partial \sigma},
\end{aligned}
$$

reemplazando (6), en la acción de Nambu-Goto (1), obtenemos:

$S=-2 \pi T_{0} \int d t R \sqrt{1-\frac{\dot{R}^{2}}{c^{2}}}$

que es la acción de Nambu-Goto (1) escrita en términos de la velocidad transversal.

\section{Ecuación dinámica}

El Lagrangiano correspondiente a nuestro sistema en estudio es, según la ecuación (7):

$$
L=-2 \pi T_{0} R \sqrt{1-\frac{\dot{R}^{2}}{c^{2}}},
$$

este depende de $R$ y $\dot{R}$, y además el sistema posee un solo grado de libertad. Haciendo uso de la ecuación de Euler-Lagrange, la ecuación de movimiento correspondiente a la cuerda bosónica circular cerrada será:

$$
R \frac{d^{2} R}{d t^{2}}-\left(\frac{d R}{d t}\right)^{2}+c^{2}=0 .
$$


La ecuación (9) se puede resolver por medio de ciertos artificios matemáticos, sin embargo el procedimiento es algo engorroso. Por otro lado si analizamos el Hamiltoniano del sistema, se podrá llegar a una solución de una manera más simple y elegante.

\section{Conservación del Hamiltoniano}

Si se considera una cuerda estirada y estática, la energía total asociada con ella no es más que el producto de la tensión de la misma por su longitud. Esto significa que si la tensión de una cuerda estática es $T_{0}$, sin importar su longitud, entonces $T_{0} a$ es la cantidad de energía que debemos utilizar para crear una cuerda de longitud $a$ [9]. Si la cuerda no permanece estática, sino que realiza movimiento, entonces debemos incluir un factor de corrección relativista. En particular para una cuerda circular de longitud $2 \pi R$, la energía total debe ser:

$$
H=\frac{2 \pi R T_{0}}{\sqrt{1-\frac{\dot{R}^{2}}{c^{2}}}},
$$

donde el denominador es el factor relativista que se debe agregar.

Se puede comprobar fácilmente la validez de la ecuación (10), pues es posible construir el Hamiltoniano del sistema a partir del Lagrangiano (8) según: $H=p \cdot v-L$.

La ecuación (10) muestra que el Hamiltoniano del sistema no depende explícitamente del tiempo, además $\frac{d H}{d t}=0$, luego el Hamiltoniano del sistema se conserva en el tiempo, así:

$H=2 \pi R_{0} T_{0}$.

El uso simultáneo de las ecuaciones (10) y (11), nos conduce a la siguiente ecuación:

$\left(\frac{d R}{d t}\right)^{2}+\left(\frac{c}{R_{0}}\right)^{2} R^{2}-c^{2}=0$.

Al derivar la ecuación (12) obtenemos:

$\frac{d R}{d t}\left[\frac{d^{2} R}{d t^{2}}+\left(\frac{c}{R_{0}}\right)^{2} R\right]=0$.

La expresión entre corchetes de la ecuación (13), es la ecuación correspondiente a un movimiento armónico simple, cuya solución se conoce y es la siguiente:

$R(t)=R_{0} \cos \left(\frac{c}{R_{0}} t\right)$

Adicionalmente, la velocidad transversal será:

$|\vec{v}|=\frac{d R}{d t}=-c \sin \left(\frac{c}{R_{0}} t\right)$.

De la ecuación (14), notamos que $R(t)=0$ para el tiempo $t=\frac{\pi R_{0}}{2 c}$.

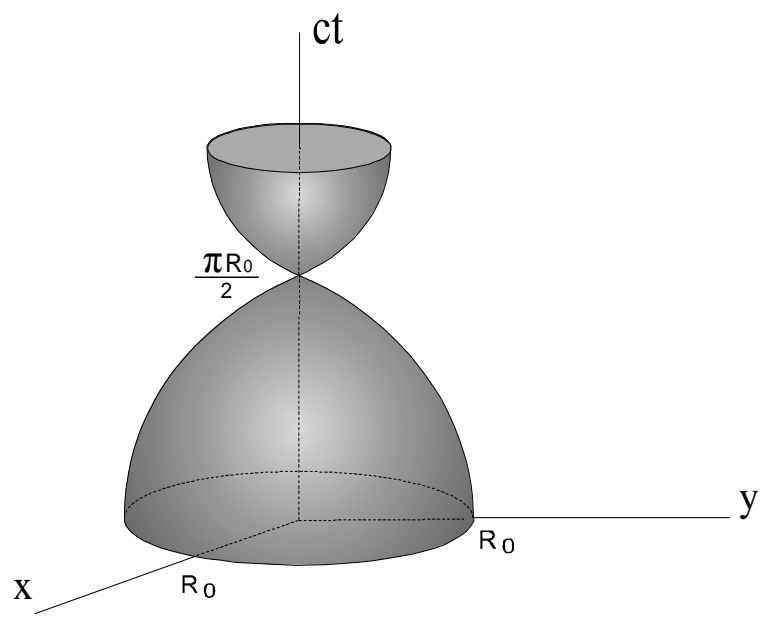

Fig. 1. Gráfica de la ecuación (14) esbozando la hoja de mundo para una cuerda bosónica circular usando un sistema coordenado tridimensional $(x, y, c t)$

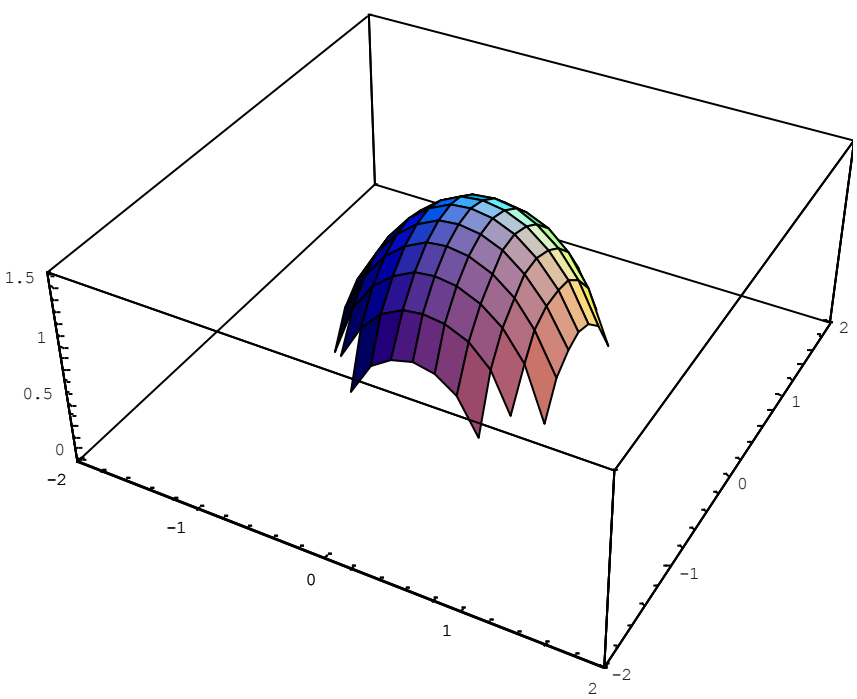

Fig.2. Gráfica de la ecuación (14) Usando el Programa Mathemática 5.1 considerando los valores: $\mathrm{R}_{0}=1,-2<x<2$, $2<y<2$. 


\section{Conclusiones}

- Las soluciones de la ecuación dinámica de la cuerda bosónica cerrada son soluciones de tipo armónico con frecuencia propia de $c / R_{0}$. Además la ecuación dinámica se resolvió usando el concepto de conservación del Hamiltoniano.

- Nótese que la ecuación (12) posee dos soluciones, una la ya hallada (ecuación 14), y la otra que corresponde al caso en que el radio de la cuerda no cambia en el tiempo. Es evidente que la solución que debemos tomar en cuenta debe satisfacer la ecuación dinámica (9), es por ello que dicha segunda solución no debe ser considerada, pues no satisface la ecuación dinámica.

\section{Referencias}

[1]. Michio Kaku, Introduction to superstring and M-theory, Springer 1999.Page 29.
[2]. M.Green, J. Schwarz, E. Witten, Superstring theory, Cambridge University Press, 1978.Page 25.

[3]. Y. Nambu, Lectures at the Copenhagen Simposium, 1970.

[4]. T. Goto, Prog. Theor. Phys., 46: 1560, 1971.

[5]. L.Brink, P. Di Vecchia, and P. Howe, Phys. Lett., B65: 471, 1976.

[6]. S.Deser and B. Zumino. Phys. Lett., B65:369, 1976.

[7]. Alexander M. Polyakov, Quantum Geometry of Bosonic String. Phys. Lett., B103: 207-210, 1981.

[8]. Lochiainn O'Raifeartaigh \& Norbert Straumann, Gauge Tjeory: Historial Origins and Some Modern Developments. 100 Years of Gravity and Accelerated Frames, World Scientific, Advanced Series on Theoretical Physics Science Vol.9 (2005).

[9]. Barton Zwiebach, A First Course in String Theory, Cambridge University Press, page 105110. 